\title{
Análise de Estratégias de Controle de Erros para Redes de Sensores com Modulação OQPSK e GFSK
}

\author{
João H. Kleinschmidt e Walter C. Borelli
}

\begin{abstract}
Resumo - Este artigo propõe um modelo analítico para cálculo da taxa de erro de pacotes e eficiência de energia de diferentes esquemas de controle de erros em redes de sensores sem fio. São usadas estratégias de retransmissão, codificação de canal e esquemas híbridos FEC/ARQ para as modulações OQPSK e GFSK. O canal sem fio é modelado com desvanecimento Rayleigh. Os resultados obtidos podem dar indicações do tipo de modulação e técnica de controle de erros a serem utilizados para determinadas condições da rede, como relação sinal-ruído e número de saltos.
\end{abstract}

Palavras-Chave - modulação, controle de erros, redes de sensores, eficiência de energia.

Abstract - This paper proposes an analytical model to evaluate the packet error rate and the energy efficiency of different error control schemes in wireless sensor networks. Retransmission strategies, channel coding and hybrid FEC/ARQ schemes for OQPSK and GFSK modulations are studied. The wireless channel is modeled with Rayleigh fading. The obtained results may indicate the modulation type and error control technique to be used in a network with different number of hops and signal-to-noise ratio values.

Index terms - modulation, error control, sensor networks, energy efficiency.

\section{INTRODUÇÃO}

As redes de sensores sem fio são um tipo especial de redes ad hoc, formada por sensores de baixo custo e baixa potência [1]. Estes dispositivos possuem componentes para sensoriamento, processamento de dados e comunicação. Algumas aplicações destas redes são o monitoramento ambiental em locais como florestas e oceanos, teste de qualidade de produtos num ambiente industrial, entre outras. Os protocolos e algoritmos (formação, roteamento, gerenciamento, etc) devem ter capacidade de autoorganização. Estas redes são caracterizadas pela limitação de energia, baixa largura de banda, pequeno tamanho de memória e capacidade computacional, grande número de nós, modelo de tráfego diferente das redes ad hoc tradicionais e sensores mais suscetíveis à falhas. $\mathrm{O}$ sensor deve enviar os dados para um dispositivo central, chamado de coletor. O nó coletor é responsável por receber todas as informações da rede, bem como executar tarefas de gerenciamento dos nós. Geralmente a rede de sensores possui múltiplos saltos, ou seja, para o sensor enviar os dados até o coletor, os dados devem passar por nós intermediários, que podem ser sensores ou outro tipo de nó com capacidade de roteamento.

João Henrique Kleinschmidt e Walter C. Borelli, Departamento de Telemática, Faculdade de Engenharia Elétrica e de Computação, Universidade Estadual de Campinas, Campinas-SP, E-mails: \{joaohk, borelli\}@dt.fee.unicamp.br.
Os canais sem fio possuem taxas de erro altas devido à interferência e ao desvanecimento gerado pelo multipercurso, levando ao desperdício de energia. Algumas técnicas podem ser empregadas para melhorar a confiabilidade dos dados enviados no canal sem fio, como o uso de estratégias de retransmissão (ARQ), códigos corretores de erro (FEC) ou o controle da potência de transmissão [2]. Embora que estratégias de controle de erros melhorem a confiabilidade de entrega de pacotes, a transmissão de bits adicionais nestes esquemas contribui também para o aumento do consumo de energia, ou seja, existe um compromisso entre a confiabilidade e o consumo de energia. Alguns autores têm estudado esta questão para esquemas de controle de erros em redes de sensores sem fio [3], [4], [5], [6], [7], [8].

Em [3] e [4] a eficiência de energia de diferentes técnicas de controle de erros foi analisada para redes de sensores com um transceptor de rádio comercial usando um modelo analítico. A confiabilidade e o consumo de energia foram analisados em [5] usando simulação para redes de sensores sem nenhuma tecnologia específica, modelo de canal ou modulação. A eficiência de energia de estratégias de controle de erros do padrão Bluetooth foi analisada em um canal com desvanecimento Rayleigh usando simulação [6] e modelo analítico [7] e as estratégias de retransmissão do padrão IEEE 802.1.4 foram comparadas em [8].

Diferente das abordagens realizadas nestes trabalhos anteriores, que utilizam uma tecnologia específica ou dispositivos comerciais, este artigo analisa as taxas de erro de pacotes e a eficiência de energia de estratégias de controle de erros para redes de sensores usando as modulações GFSK (Gaussian Frequency-Shift Keying) e OQPSK (Offset Quadrature Phase-Shift Keying). Estas modulações são utilizadas nos padrões para redes pessoais sem fio IEEE 802.15.1 e IEEE 802.15.4, respectivamente. Estes padrões de redes sem fio foram projetados especificamente para redes com restrições de energia. São analisados sistemas com e sem retransmissão, além de dois tipos de códigos corretores de erro: Hamming e $\mathrm{BCH}$. Algumas estratégias de controle de erros para redes de sensores sem fio são apresentadas na Seção II. A Seção III descreve um modelo analítico para cálculo de probabilidade de erro de pacotes e eficiência de energia. Os resultados obtidos são descritos na Seção IV e a Seção V faz as considerações finais.

\section{CONTROLE DE ERROS PARA REDES DE SENSORES}

Os requisitos de consumo de energia, a estrutura de múltiplos saltos e a transmissão broadcast do canal sem fio fazem com que as redes de sensores sem fio necessitem de diferentes tipos de estratégias de controle de erros. Diferentes 
parâmetros de desempenho, como consumo de energia, latência e taxa de erro de pacotes devem ser analisados.

A codificação de canal usa códigos corretores de erros para combater erros de bit adicionando redundância (bits de paridade) aos pacotes de dados antes que sejam transmitidos. Esta redundância é usada pelo receptor para detectar e corrigir erros. Sistemas ARQ têm apenas a capacidade de detectar erros e não é feita nenhuma tentativa para corrigir os pacotes recebidos com erro; é feita uma requisição para que os pacotes recebidos com erro sejam retransmitidos. A detecção de erros é geralmente feita usando um código CRC (Cyclic Redundancy Check).

FEC e ARQ são as duas categorias básicas de técnicas de controle de erros. ARQ é relativamente simples e tem bom desempenho se as taxas de erro não são muito grandes. No entanto, pode levar a grandes atrasos na rede se as condições de canal são ruins. Os esquemas FEC têm um bom desempenho e podem manter as taxas de atraso constantes mesmo em más condições, mas para manter a confiabilidade vários padrões de erro devem ser corrigidos. Então um código muito longo é necessário, impondo uma sobrecarga de transmissão bastante alta. Outro fator é que o canal sem fio é não estacionário e as taxas de erro de bit variam com o tempo. Os códigos são estacionários e devem ser implementados para garantir um determinado desempenho para o pior caso das características do canal. Logo, são associadas a uma sobrecarga de bits desnecessária quando o canal está em boas condições.

Para melhorar as desvantagens de sistemas FEC e ARQ, a combinação desses dois esquemas pode ser feita, os chamados esquemas ARQ híbridos. Neste trabalho são usados esquemas híbridos do tipo I, que inclui bits de paridade tanto para detecção como para correção de erros. Se o número de bits com erro pode ser corrigido pelo código não é solicitada retransmissão. Se um erro não corrigível é detectado, o receptor descarta o pacote e solicita a retransmissão. Este processo é repetido até que a palavra código seja recebida corretamente ou o número máximo de retransmissões seja atingido.

Neste artigo são propostos e analisados quatro esquemas de controle de erros:

1) código CRC para detecção de erros sem retransmissão;

2) código CRC para detecção de erros com retransmissão.

3) codificação de canal com código CRC para detecção de erros sem retransmissão.

4) codificação de canal com código CRC para detecção de erros com retransmissão.

No primeiro esquema o código CRC é usado apenas para verificação de erros. Se houver erro o pacote é descartado e não é transmitido para o próximo nó (numa rede com múltiplos saltos). Num esquema sem CRC, o pacote seria transmitido ao próximo nó mesmo que tivesse erros. No segundo esquema, o código CRC é usado para detecção de erros e solicitação de retransmissão. É enviado um pacote para confirmar ou não a recepção, fazendo com que o transmissor reenvie o pacote de dados em caso de erros. $\mathrm{O}$ terceiro e quarto esquemas funcionam como os dois primeiros, com a diferença que usam adicionalmente um código corretor de erros (Hamming ou $\mathrm{BCH}$ ). Estes quatro esquemas são comparados com um sistema sem capacidade de detecção/correção de erros ou retransmissão.

O pacote de dados da camada de enlace é a unidade de comunicação entre os nós sensores da rede, consistindo de um cabeçalho de $c$ bits, um trailer de $t$ bits e $d$ bits de dados, como mostra a Figura 1. O pacote de retorno no caso de retransmissão possui o mesmo formato, porém sem o campo para dados. A Tabela I mostra os tipos de pacotes utilizados neste trabalho, com as informações sobre o controle de erros utilizado, modulação e tamanho dos dados.

\begin{tabular}{|c|c|c|}
\hline $\begin{array}{c}\text { cabeçalho } \\
\text { c bits }\end{array}$ & $\begin{array}{c}\text { dados } \\
\text { d bits }\end{array}$ & $\begin{array}{c}\text { trailer } \\
\text { t bits }\end{array}$ \\
\hline
\end{tabular}

Fig. 1. Formato do pacote.

Tabela I. Tipos de pacotes.

\begin{tabular}{|c|c|c|c|c|c|}
\hline Tipo & Modulação & ARQ & CRC & FEC & $\begin{array}{c}\text { Dados } \\
\text { (bytes) }\end{array}$ \\
\hline NO3/2 & OQPSK & Não & Não & Não & $32 / 256$ \\
\hline CO3/2 & OQPSK & Não & Sim & Não & $32 / 256$ \\
\hline AO3/2 & OQPSK & Sim & Sim & Não & $32 / 256$ \\
\hline HO3/2 & OQPSK & Sim & Sim & Hamming & $32 / 256$ \\
\hline BO3/2 & OQPSK & Sim & Sim & BCH & $32 / 256$ \\
\hline OB3/2 & OQPSK & Não & Sim & BCH & $32 / 256$ \\
\hline NG3/2 & GFSK & Não & Não & Não & $32 / 256$ \\
\hline CG3/2 & GFSK & Não & Sim & Não & $32 / 256$ \\
\hline AG3/2 & GFSK & Sim & Sim & Não & $32 / 256$ \\
\hline HO3/2 & GFSK & Sim & Sim & Hamming & $32 / 256$ \\
\hline BG3/2 & GFSK & Sim & Sim & BCH & $32 / 256$ \\
\hline CB3/2 & GFSK & Não & Sim & BCH & $32 / 256$ \\
\hline
\end{tabular}

\section{MODELO ANALÍTICO}

\section{A. Cálculo da probabilidade de erro de pacote}

Um pacote enviado não é aceito pelo receptor quando qualquer um dos cinco eventos acontece: (A) o cabeçalho do pacote enviado está corrompido; (B) o destino falha ao sincronizar com o trailer do pacote enviado; (C) os dados do pacote enviado estão corrompidos, causando a falha na verificação de CRC; (D) o cabeçalho do pacote de retorno está corrompido e (E) a fonte é incapaz de sincronizar com o trailer do pacote de retorno. Está sendo assumido que os erros são estatisticamente independentes.

O cabeçalho é recebido corretamente se todos os bits são recebidos corretamente:

$$
P\left[\overline{A]}=\left[1-p\left(\gamma_{f}\right)\right]^{c},\right.
$$

onde $p\left(\gamma_{f}\right)$ é a probabilidade de erro de bit do canal direto em função da relação sinal-ruído (SNR) média recebida $\bar{\gamma}$. Como o pacote de retorno ACK também tem um cabeçalho de $c$ bits, a probabilidade para o evento $\mathrm{D}$ tem a mesma forma,

$$
P[\bar{D}]=\left[1-p\left(\gamma_{r}\right)\right]^{c},
$$

onde $p\left(\gamma_{r}\right)$ é a probabilidade de erro de bit do canal reverso. Os eventos B ou E ocorrem se qualquer bit do trailer de sincronização for recebido com erro:

$$
P\left[\overline{B]}=\left[1-p\left(\gamma_{f}\right)\right]^{t}\right.
$$




$$
P[\bar{E}]=\left[1-p\left(\gamma_{r}\right)\right]^{t}
$$

O erro mais provável é o do tipo $\mathrm{C}$, que ocorre quando os bits de dados são recebidos com erros. Para o caso sem nenhum tipo de código corretor de erros:

$$
P[\bar{C}]=\left[1-p\left(\gamma_{f}\right)\right]^{d},
$$

Para pacotes em que os dados são protegidos com um código corretor de erros, a probabilidade do evento $\mathrm{C}$ é calculada considerando a capacidade de correção do código. Para um código de Hamming de taxa 2/3 $(15,10)$, capaz de corrigir todos os padrões de erros simples e detectar todos os erros duplos numa palavra código, tem-se que:

$$
P[\bar{C}]=\left[15 p\left(\gamma_{f}\right)\left[1-p\left(\gamma_{f}\right)\right]^{14}+\left[1-p\left(\gamma_{f}\right)\right]^{15}\right]^{B},
$$

onde $B$ é o número de blocos de 10 bits que entram no codificador. Outro código utilizado neste trabalho é um código $\mathrm{BCH}(511,259,30)$. A probabilidade do evento $\mathrm{C}$ neste caso é:

$$
P[\bar{C}]=\sum_{k=0}^{30}\left(\begin{array}{c}
511 \\
k
\end{array}\right) \cdot\left[p\left(\gamma_{f}\right)\right]^{k} \cdot\left[1-p\left(\gamma_{f}\right)\right]^{511-k}
$$

Neste artigo são consideradas as modulações OQPSK e GFSK. A probabilidade de erro de bit $p(\gamma)$ varia para cada tipo de modulação. Para a modulação OQPSK é dada por [9]:

$$
p(\gamma)=Q(\sqrt{2 \gamma})
$$

onde $Q(x)$ é :

$$
Q(x)=\frac{1}{\sqrt{2 \pi}} \int_{x}^{\infty} \exp \left(\frac{-u^{2}}{2}\right) d u
$$

Para a modulação GFSK com produto tempo-largura de faixa $\mathrm{BT}=0,5$ e índice de modulação entre 0,28 e 0,35, a probabilidade de erro de bit $p(\gamma)$ é calculada da seguinte forma [10]:

$$
p(\gamma)=Q_{1}(a, b)-\frac{1}{2} e^{\left(a^{2}+b^{2}\right) / 2} I_{o}(a b)
$$

Sendo que $Q_{1}(a, b)$ é a função Q-Marcum, $I_{o}$ é a função de Bessel modificada de primeira ordem e $a$ e $b$ são constantes que dependem da relação sinal-ruído, da correlação $\rho$ dos sinais e do índice de modulação $i$, dados pelas equações (11) e (12). Neste trabalho foi utilizado um índice de modulação $i=0,32$.

$$
\begin{gathered}
\rho=\frac{\sin (2 \pi i)}{2 \pi i} \\
a=\sqrt{\frac{\gamma}{2}\left(1-\sqrt{1-\rho^{2}}\right)}, b=\sqrt{\frac{\gamma}{2}\left(1+\sqrt{1-\rho^{2}}\right)}
\end{gathered}
$$

Assim, a probabilidade de erro de pacote do canal direto, $P E R_{f}$, e reverso, $P E R_{r}$, podem ser definidas por:

$$
\begin{aligned}
P E R_{f} & =1-\int_{0}^{\infty} f\left(\gamma_{f}\right) P\left[\overline { A ] } P \left[\overline{B]} P[\bar{C}] d \gamma_{f}\right.\right. \\
P E R_{r} & =1-\int_{0}^{\infty} f\left(\gamma_{r}\right) P[\bar{D}] P[\bar{E}] d \gamma_{r},
\end{aligned}
$$

onde $f\left(\gamma_{f}\right)$ e $f\left(\gamma_{r}\right)$ são as funções de densidade de probabilidade do canal direto e reverso, respectivamente.

O canal sem fio é modelado usando desvanecimento Rayleigh. Esta distribuição é amplamente utilizada para a modelagem de canais sem fio. A função densidade de probabilidade é dada por:

$$
f(\gamma)=\frac{1}{\bar{\gamma}} \exp \left(-\frac{\gamma}{\bar{\gamma}}\right), \quad \text { para } \gamma \geq 0
$$

onde $\bar{\gamma}$ é a SNR media recebida e $\gamma$ é a SNR instantânea. As probabilidades de erro de pacote podem então ser calculadas usando a equação (15) em (13) e (14). Está sendo considerado que as condições de propagação entre o transmissor e o receptor são as mesmas nas duas direções, ou seja, $\gamma_{f}=\gamma_{r}$.

A probabilidade de um pacote ser corretamente recebido é a probabilidade de sucesso do pacote nos canais direto e reverso. Assim, a probabilidade de erro de pacote $P E R$ é dada por:

$$
P E R=1-\left[\left(1-P E R_{f}\right)\left(1-P E R_{r}\right)\right]
$$

\section{B. Cálculo da eficiência de energia}

A probabilidade de um pacote ser corretamente recebido no nó coletor para os pacotes sem ARQ é dada por:

$$
P_{\text {narq }}=\left(1-P E R_{f}\right)^{H}
$$

onde $H$ é o número total de saltos. Considere $n$ o número de retransmissões de um pacote com ARQ. Assumindo que o código CRC é capaz de detectar todos os erros e número máximo de retransmissões infinito, a probabilidade que um pacote chegue corretamente no coletor é:

$$
P_{\text {arq }}=\sum_{n=0}^{\infty}\left[\left(1-P E R_{f}\right)\left(1-P E R_{r}\right)\right]^{H+n}=1
$$

A probabilidade de acontecerem $n$ retransmissões é o produto de falha nas $n-1$ transmissões e a probabilidade de sucesso na enésima transmissão:

$$
p_{N}[n]=(1-P E R)(P E R)^{n-1}
$$

Então, a equação (20) é usada para calcular o número médio de retransmissões $\bar{N}$ em um salto:

$$
\bar{N}=\sum_{n=1}^{\infty} p_{N}[n] \times n
$$

O número de pacotes recebidos com erro no coletor pode ser definido para os pacotes sem ARQ como o produto do número total de pacotes transmitidos $n_{p a c}$ e a probabilidade que o pacote chegue com erro no coletor:

$$
n_{\text {error }}=\left(1-P_{\text {narq }}\right) \times n_{\text {pac }}
$$

Fazendo as mesmas considerações da equação (18), nenhum dos pacotes ARQ é recebido no coletor com erros e assim $n_{\text {error }}=0$ :

$$
n_{\text {error }}=\left(1-P_{a r q}\right) \times n_{p a c}=0
$$

A confiabilidade $R$ é dada pela porcentagem dos pacotes enviados que são recebidos corretamente no coletor e pode ser calculada da seguinte forma:

$$
R=\left[\left(n_{p a c}-n_{\text {error }}\right) / n_{p a c}\right]
$$

Como não está sendo usado nenhum hardware específico, o consumo de energia é expresso de forma normalizada. As energias consideradas são as gastas no processo de comunicação (transmissão e recepção). 
É considerado o mesmo modelo de [4] e [5], onde a recepção de um bit consome aproximadamente $75 \%$ da energia gasta para transmitir um bit. A energia mínima consumida $E_{\min }$ para $H$ saltos é calculada para um pacote sem nenhum controle de erros (sem ARQ):

$$
E_{\text {min }}=H \times n_{\text {pac }} \times\left(n_{\text {bits }}+n_{\text {bits }} \times 0.75\right),
$$

onde $n_{\text {bits }}$ é o número total de bits do pacote. A energia consumida total $E$ numa rede de sensores para um pacote sem ARQ é o número total de bits transmitidos e recebidos:

$$
E=H \times n_{\text {pac }} \times\left(n_{\text {bits }}+n_{\text {bits }} \times 0.75\right)
$$

Para os pacotes com ARQ, a energia $E$ é o número total de bits transmitidos e recebidos, incluindo as retransmissões:

$$
E=H \times n_{\text {pac }} \times \bar{N} \times\left[n_{\text {bits }}+n_{\text {ack }}+\left(n_{\text {bits }}+n_{\text {ack }}\right) \times 0.75\right],
$$

onde $n_{a c k}$ é o número total de bits do pacote de retorno (ACK).

Para calcular a energia $E$ para o caso de código CRC sem ARQ é preciso calcular o número médio de saltos que um pacote vai ter na rede sem ser descartado. A probabilidade que um pacote atinja $h$ saltos é o produto do sucesso em $h-1$ saltos e a probabilidade de falha no h-ésimo salto, se $h<H$. Se $h=H$ a probabilidade de um pacote atingir $H$ saltos é o produto do sucesso nos $h-1$ saltos e a probabilidade de falha no h-ésimo salto mais a probabilidade de sucesso em $H$ saltos:

$$
\begin{gathered}
p_{H}[h]=\left[\left(1-P E R_{f}\right)^{h-1}\left(P E R_{f}\right)\right], \text { se } \mathrm{h}<\mathrm{H} \\
p_{H}[h]=\left[\left(1-P E R_{f}\right)^{h-1}\left(P E R_{f}\right)+\left(1-P E R_{f}\right)^{h}\right], \text { se } \mathrm{h}=\mathrm{H}
\end{gathered}
$$

Então o número médio saltos $\bar{H}$ pode ser calculado:

$$
\bar{H}=\sum_{h=1}^{H} p_{H}[h] \times h
$$

A energia total consumida $E$ para os pacotes com CRC e sem ARQ é:

$$
E=\bar{H} \times n_{\text {pac }} \times\left(n_{\text {bits }}+n_{\text {bits }} \times 0.75\right)
$$

Para uma rede de sensores ser considerada eficiente em energia, o máximo número de bits de dados deve ser transmitido com o mínimo consumo de energia. O parâmetro de eficiência de energia $\eta$ pode ser definido como:

$$
\eta=\frac{E_{\min }}{E} \times R
$$

\section{RESULTADOS}

As Figuras 2 a 7 mostram os resultados obtidos usando o modelo analítico de probabilidade de erro de pacotes e eficiência de energia para os diferentes pacotes e esquemas de controle de erros apresentados na Seção II. O número de bits utilizados para o cabeçalho do pacote foi $c=32$ e para o trailer $t=8$ bits.

A Figura 2 mostra a taxa de erro de pacotes $P E R_{f}$ em função da relação sinal-ruído. São comparadas as modulações OQPSK e GFSK com e sem codificação. A $P E R_{f}$ da modulação GFSK é sempre mais alta que a da modulação OQPSK, pois a probabilidade de erro de bit também é maior [9]. Quando comparado um pacote sem codificação com outro que usa código corretor de erros, a $P E R_{f}$ de um pacote codificado é sempre mais baixa. O código de Hamming tem um melhor desempenho para as duas modulações quando comparado ao sistema não codificado. Por corrigir mais erros, o código $\mathrm{BCH}$ apresenta um desempenho ainda melhor para a taxa de erro de pacotes.

Nos resultados obtidos para eficiência de energia das Figuras 3 a 7 um sensor envia 100000 pacotes ao coletor $\left(n_{p a c}=100000\right)$. Apenas um nó sensor transmite de cada vez. A Figura 3 compara os esquemas de controle de erros 1 e 2 da Seção II para as duas modulações numa rede com 15 saltos e dados de 32 bytes. Com este número de saltos $(H=15)$ o desempenho relativo entre os pacotes tende a estabilizar [6], [7]. Como esperado, a eficiência da modulação OQPSK é melhor que da GFSK quando comparadas às mesmas estratégias de controle de erros (AO3 e AG3, por exemplo). Quando analisado o desempenho dos pacotes somente da modulação OQPSK, o pacote CO3 é melhor para valores altos de SNR e o pacote AO3 para valores menores (abaixo de 25 $\mathrm{dB}$, aproximadamente). A mesma conclusão é válida para a modulação GFSK, mudando apenas o valor que o pacote CG3 é mais eficiente em energia que o pacote AG3 (acima de 30 $\mathrm{dB}$, aproximadamente).

As Figuras 4 e 5 mostram a eficiência de energia para 2 saltos e 32 bytes de dados para OQPSK e GFSK, respectivamente. Com poucos saltos a melhor opção em uma ampla faixa de relação sinal-ruído é usar código CRC para detecção de erros, porém sem retransmissão (CO3 e CG3). Apenas para valores baixos de SNR usar código $\mathrm{BCH}$ é a melhor opção, porém também sem retransmissão (OB3 e CB3). A diferença entre os dois esquemas de modulação é que para GFSK o código BCH é mais eficiente para valores de SNR abaixo de $10 \mathrm{~dB}$ (Figura 5), enquanto que para OQPSK torna-se eficiente apenas para valores abaixo de $5 \mathrm{~dB}$ (Figura 4).

Quando o número de bytes de dados do pacote aumenta, algumas mudanças podem ser verificadas, como mostram as Figuras 6 e 7 . Com 256 bytes de dados usar retransmissão sem código é a estratégia mais eficiente para valores de SNR acima de 5 dB (OQPSK - Figura 6) ou $10 \mathrm{~dB}$ (GFSK - Figura 7). Usar codificação de canal só e mais eficiente para valores baixos de SNR, enquanto que usar código CRC sem retransmissão ( $\mathrm{CO} 2$ ou $\mathrm{CG} 2)$ não possui melhor eficiência de energia para nenhum valor de SNR. O principal fator que leva ao desperdício de energia em sistemas ARQ são os bits adicionais enviados no pacote de retorno usado para confirmar ou não o recebimento de um pacote. Como o número de bits deste pacote de retorno é fixo, quando o tamanho dos dados aumenta, o impacto do número de bits do pacote de retorno já não é tão significativo, fazendo com que a eficiência de energia aumente.

Nos resultados obtidos pode-se observar que para cada condição de canal, número de saltos e tamanho de dados uma determinada técnica de controle de erros apresenta um melhor desempenho. Usar codificação de canal é a melhor opção apenas para valores baixos de SNR e poucos saltos ou também SNR baixa e tamanho de dados grande. As estratégias de controle de erros têm um comportamento semelhante para as duas modulações estudadas, porém o impacto de erros é sempre maior na modulação GFSK, que precisa de um valor de SNR um pouco maior que da modulação OQPSK para atingir o mesmo desempenho. 


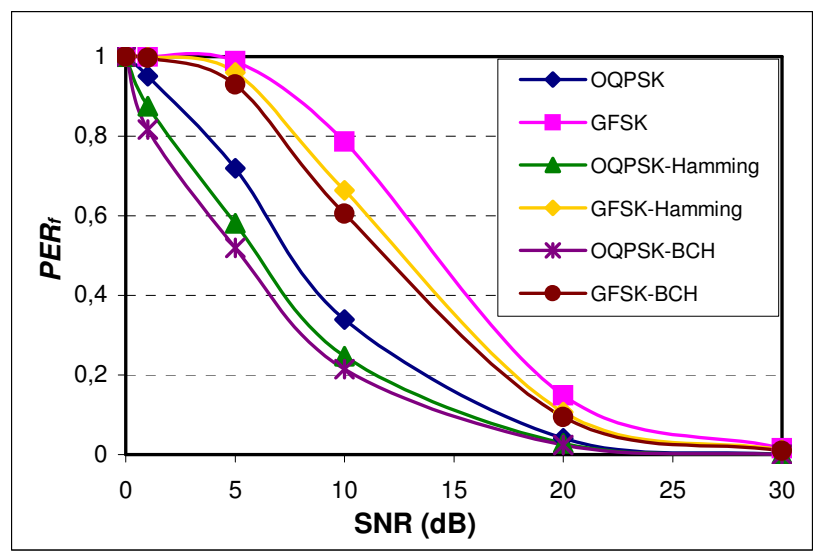

Fig. 2. Probabilidade de erro de pacote, 32 bytes.

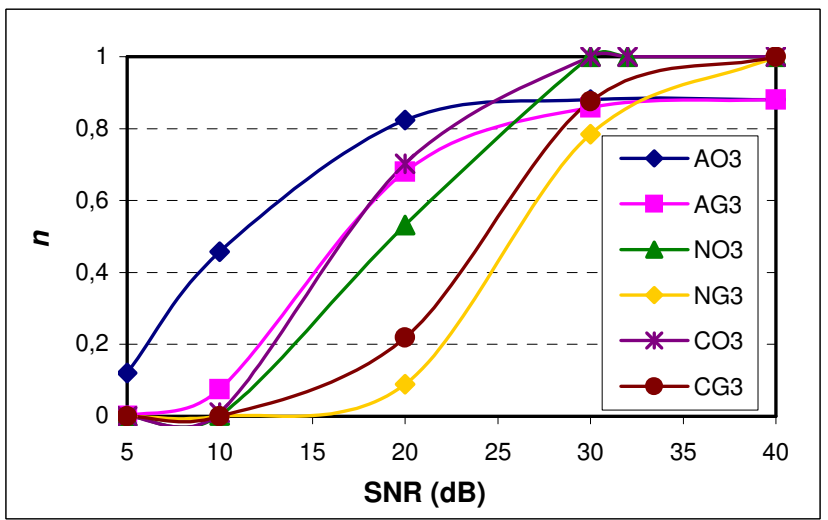

Fig. 3. Eficiência de energia para 15 saltos, 32 bytes.

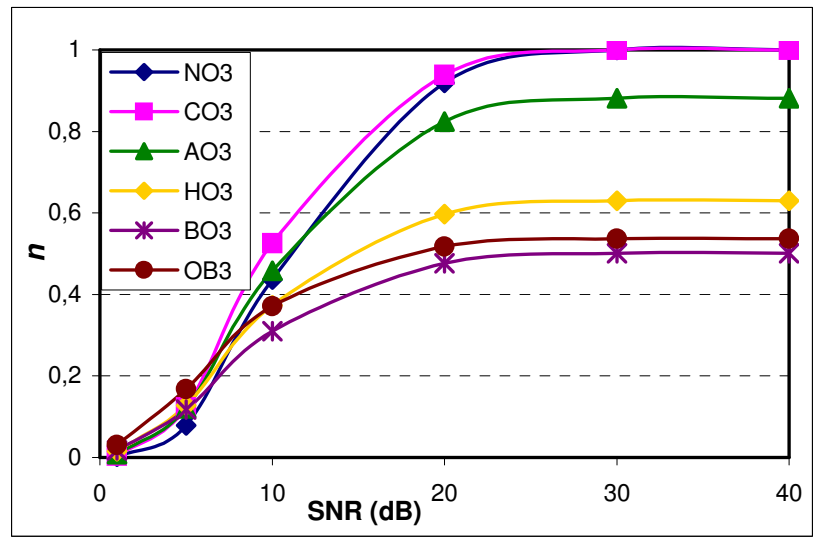

Fig. 4. Eficiência de energia para 2 saltos, 32 bytes, modulação OQPSK.

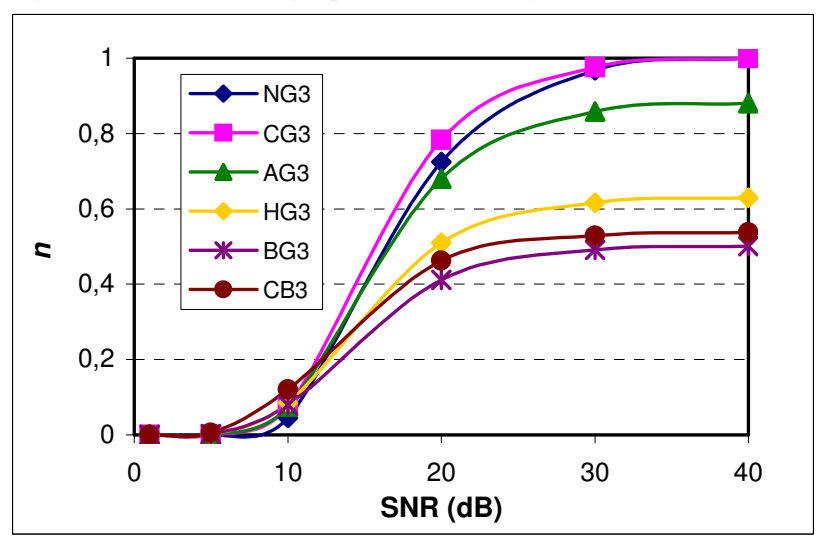

Fig. 5. Eficiência de energia para 2 saltos, 32 bytes, modulação GFSK.

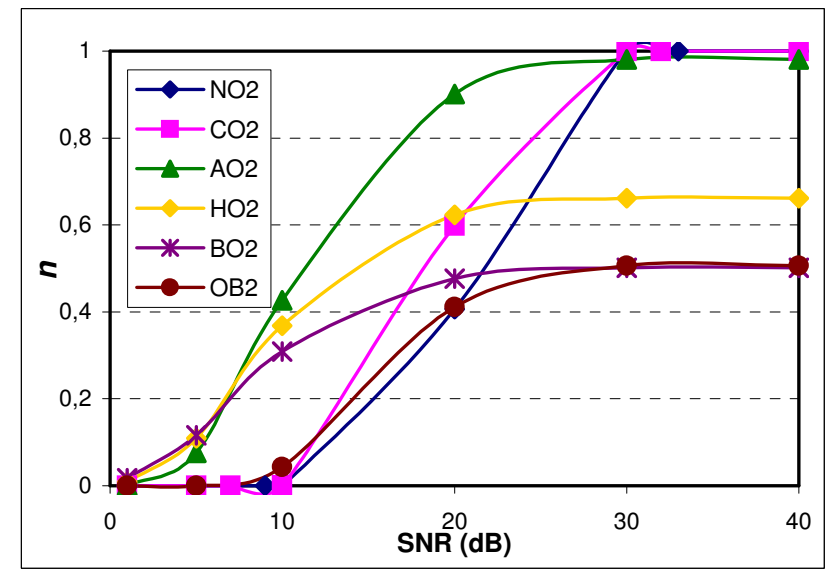

Fig. 6. Eficiência de energia para 15 saltos, 256 bytes, modulação OQPSK.

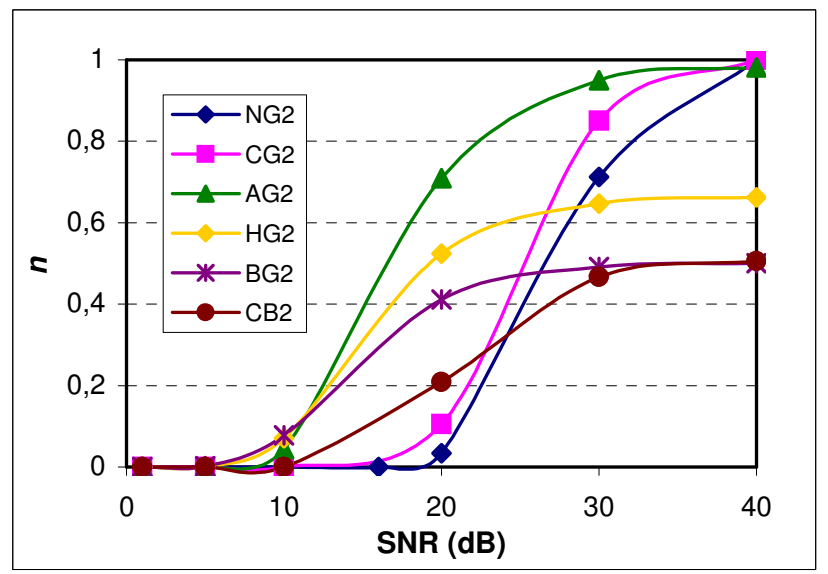

Fig. 7. Eficiência de energia para 15 saltos, 256 bytes, modulação GFSK.

\section{CONCLUSÃO}

Neste artigo foram propostas e analisadas diferentes estratégias de controle de erros para redes de sensores sem fio para as modulações OQPSK e GFSK. As técnicas estudadas incluem códigos $\mathrm{CRC}$, retransmissão (ARQ), códigos de Hamming e $\mathrm{BCH}$ e técnicas híbridas FEC/ARQ. Um modelo analítico foi apresentado para cálculo de probabilidade de erro de pacotes e da métrica de eficiência de energia em canais com desvanecimento Rayleigh. A modulação OQPSK mostrou-se mais eficiente em energia do que a modulação GFSK, pois tem menor probabilidade de erro de símbolo. Os códigos corretores de erros utilizados apresentam bom desempenho para diminuir a probabilidade de erro de pacote, porém são eficientes em energia apenas para baixos valores de relação sinal-ruído. Usar código CRC para detecção de erros sem retransmissão mostrou-se eficiente para altos valores de SNR e poucos saltos. Usar retransmissão é a melhor opção para redes com muitos saltos e/ou quando o pacote possui um grande número de bytes de dados. Os resultados obtidos podem dar indicações do tipo de modulação e estratégia de controle de erros a ser utilizada numa aplicação de sensoriamento. $\mathrm{O}$ modelo apresentado neste artigo pode ser adaptado para outras modulações e esquemas de controle de erros. 


\section{REFERÊNCIAS}

[1] I. F. Akyildiz, W. Su, Y. Sankarasubramaniam e E. Cayirci, "A survey on sensor networks", IEEE Communications Magazine, pp. 102-114, Agosto 2002.

[2] H. Liu, H. Ma, M. El Zarki e S. Gupta, "Error control schemes for networks: an overview", Mobile Networks and Applications, vol. 2, pp. 167-182, Junho 1997.

[3] Y. Sankarasubramanian, I. F. Akyildiz e S. W. Mc Laughlin, "Energy efficiency based packet size optimization in wireless sensor networks", Proc. of Sensor Network Protocols and Applications, 2003.

[4] H. Karvonen, Z. Shelby e C. Pomalaza-Ráez. "Coding for energy efficient wireless embedded networks", Int. Workshop on Wireless Ad-hoc Networks, 2004.

[5] J. Meer, M. Nijdam e M. Bijl, "Adaptive error control in a wireless sensor network using packet importance valuation", Hardware/software codesign, Enschede, Holanda, Maio 2003.

[6] J. H. Kleinschmidt, W. C. Borelli e M. E. Pellenz, "Custom error control schemes for energy efficient Bluetooth sensor networks", SBrT/IEEE International Telecommunications Symposium, Fortaleza, Brasil, Setembro 2006.

[7] J. H. Kleinschmidt, W. C. Borelli e M. E. Pellenz, "An analytical model for energy efficiency of error control schemes in sensor networks", IEEE International Conference on Communications, Glasgow, Escócia, Junho 2007.

[8] J. H. Kleinschmidt e W. C. Borelli, "Análise da eficiência de energia de estratégias de retransmissão em redes de sensores IEEE 802.15.4", $5^{\text {th }}$ International Telecommunications and Technologies Symposium, Cuiabá- MT, Brasil, Dezembro 2006.

[9] B. Sklar, Digital Communications, Prentice Hall, 1995.

[10] J. Proakis, Digital Communications, New York, NY: McGraw-Hill, $4^{\text {th }}$ edition, 2001. 